\title{
Context-Aware Multimedia Content Adaptation for Mobile Web
}

\author{
Dongsong Zhang \\ Department of Information Systems, University of Maryland, Baltimore County \\ 1000 Hilltop Circle, Baltimore, Maryland, 21250, USA \\ Email: zhangd@umbc.edu
}

\author{
Anil Jangam \\ Department of Computer Science, University of Maryland, Baltimore County \\ 1000 Hilltop Circle, Baltimore, Maryland, 21250, USA \\ Email: anilj1@umbc.edu

\section{Lina Zhou} \\ Department of Information Systems, University of Maryland, Baltimore County \\ 1000 Hilltop Circle, Baltimore, Maryland, 21250, USA \\ Email: zhoul@umbc.edu

\section{Isil Yakut} \\ Department of Information Systems, University of Maryland, Baltimore County \\ 1000 Hilltop Circle, Baltimore, Maryland, 21250, USA \\ Email: yakut1@umbc.edu
}

\begin{abstract}
Multimedia content in web pages poses a variety of problems to mobile web users, such as incompatibility with mobile devices or failing to meet accessibility needs of users with vision disabilities. To address those problems, this study proposes and evaluates a context-aware approach to multimedia content adaptation for mobile Web. It uniquely takes into consideration not only users' preferences and accessibility needs, but also capabilities of multimedia support of specific mobile devices. Evaluation shows that the proposed approach is accurate and efficient. This study provides insights for research on context-aware mobile computing, adaptive interfaces, and mobile HCI.
\end{abstract}

Keywords: Context-awareness, user-centered, multimedia content; adaptation; mobile devices 


\section{Introduction}

In recent years, there has been remarkable growth of the use of smart phones featured with relatively high processing power, diverse sensors, and strong multimedia-support capabilities. The proliferation and advancement of those portable mobile handheld devices and wireless communication technology offer an unprecedented level of flexibility, accessibility, and convenience to users and open new venues and possibilities for rich context-aware mobile applications, particularly for ubiquitous information access. ${ }^{1}$ Mobile phones have become an integral part of many people's daily life. According to the International Telecommunication Union, there were 6.8 billion mobile phone users worldwide at the end of 2012 (http://mobithinking.com/mobile-marketing-tools /latest-mobile-stats/a), which is equivalent to $96 \%$ of the entire world population and a big leap from 5.4 billion mobile phone users in 2010 .

Despite continuous improvement in the past decade, the convenience and effectiveness of mobile web (i.e., web browsing through mobile devices) have been compromised by various constraints of mobile phones and wireless networks. ${ }^{2}$ In particular, multimedia content in websites may pose a variety of problems to mobile web users. First, not every media format is supported by all mobile phones. It will be meaningless and a waste of time and network bandwidth to transmit a video or audio file in a website to a mobile phone if the file format is incompatible with the phone. Second, users may have different needs for adaptation of multimedia content. For example, a blind user may not need the visual signal of video or even entire video to be transmitted to his/her device. Third, the original display size of video or image files may be larger than the maximum width and length that a mobile device can display in one screen and therefore should be re-scaled to fit into the screen of the device. Therefore, multimedia adaptation is vital for improving not only the functioning of wireless networks, but also user experience.

So far, there has been relatively little research that aims to address those problems in mobile Web. The adaptation of multimedia content according to context information is essential to multimedia streaming applications. ${ }^{3}$ In particular, dynamic adaptation based on real-time context can be very useful and practical for interactive multimedia applications, because it allows the delivery of highly customized multimedia content to the end user without the need of generating multiple transcoded versions in advance. ${ }^{4}$ The term context is defined as "any real-time information or entity that influences interaction between users and mobile applications, as well as potentially changes the preferred behavior of those applications (p.29) ${ }^{5}$ ". Context awareness is the ability of an application to detect and understand its situational context and to adapt its behavior in a user preferred manner accordingly. ${ }^{5}$

This research proposes a user-centered, device-aware, and dynamic multimedia adaptation approach for mobile Web. Mobile web adaptation refers to the change of content and/or display of web pages in order to make web browsing on mobile handheld devices more effectively and accessible. ${ }^{2}$ The proposed method is aimed to adapt multimedia content in web pages based on users' specific needs and characteristics of mobile phones (i.e., context). User-centered adaptation means that adaptation will be driven by the needs and preferences of individual users. Our proposed method automatically adapts multimedia content in a web page at run time to not only address the accessibility needs and personal adaptation preferences of users (i.e., user context), but also take multimedia support capabilities of individual mobile devices (i.e., device context) into consideration. The initial performance evaluation shows that the proposed approach is accurate and efficient. By incorporating user and device context, our proposed approach makes important contributions to the research fields of mobile HCI, adaptive user interface, and context-aware mobile systems.

The rest of the paper will be organized as follows. We will first introduce the related work on multimedia adaptation in Section 2. Then, we will present our approach to context-aware multimedia adaptation for mobile Web in Section 3, followed by the introduction to evaluation and results in Section 4. Finally, we conclude the paper with future research directions in Section 5.

\section{Related Work}

With the frequent introduction of new mobile devices to the market, the number and diversity of mobile devices are constantly growing. Such diversities have changed the logic behind web content authoring. In the meantime, web content is richer and using various formats. Therefore, how to adapt diverse multimedia content in web pages to fit individual mobile devices becomes essential.

There has been increasing research on multimedia adaptation in the past decade, particularly on video adaptation. ${ }^{2}$ Kim et al. propose a context-aware adaptive streaming framework for transmitting multimedia content in mobile web environments that manages 
multimedia content inputs from multimedia sensors. $^{3}$ It controls multimedia content quality based on various context information, such as content, device, location, user, time, and network. However, this framework is not designed for multimedia adaptation for mobile Web, and authors have not performed any evaluation.

Generally, there are two types of video adaptation, namely structural and semantic adaptation. In structural adaptation, video content is adapted to the capabilities of network constraints. For example, the bit-rate or resolution of video can be reduced to meet the requirements of transmission. As a result, structural adaptation techniques may affect the quality of video. In the study of Tian and Liu, ${ }^{6}$ video content is segmented into small chunks (seconds or tens of seconds), which can be encoded in several different bit rates. The transmission of individual video chunks will be decided at run time in reaction to the network congestion condition. Concerns with their approach are that it reacts slowly to the change of network congestion conditions and is very sensitive to variations in network bandwidth. Tian and Liu propose to use a correction mechanism based on client-side buffered video to achieve a smooth adaptation of video. ${ }^{6}$ They use a machinelearning based profiling of TCP congestion to predict TCP throughput based on historical data.

Semantic video adaptation can be achieved through annotating regions of interests (ROI) in video frames. Different adaptation priorities can be assigned to different portions of video. ${ }^{7,8}$ The limitation of this line of approach lies in its difficulty in correctly identifying ROIs. To perform semantic adaptation, automatic video content analysis either by annotation or by cropping and scaling certain regions of video is required. Bruyne et al. propose an approach that performs semantic analysis and adaptation at a presentation layer. ${ }^{7}$ In another study, researchers propose a mechanism to process video content based on semantic preferences set by users. ${ }^{8}$ Video is viewed as a set of semantic entities, with each having some relationship with others. In the adaptation process, semantic entities of video will be modified or removed (e.g., remove violent content from video).

Kofler et al. use recent advancements in scalable video coding schemes that help reduce the complexities of real-time video adaptation. ${ }^{9}$ The layered video encoding can discard certain layers of a video stream, which makes it possible to extract variations of video with different spatial resolutions, frame rates, and bit rates. As different components of video files can now be processed independently, it is possible to process video content on a resource-limited platform.

Some existing research on image adaptation for mobile devices focuses on identifying the importance of images or regions of an image interesting to users and then adapting those identified images or image regions through methods such as transcoding. Transcoding is the process by which a data object in one representation is converted into another one. For example, Yin et al. propose a semantics-based consumer photo adaptation scheme for users of mobile devices. ${ }^{10}$ They integrate semantic information provided by users with low level image features using a Bayesian fusion approach to identify semantically important regions of interest. Then, they perform adaptation of perceptually important ROI of each photo to fit small displays of mobile devices. Some studies propose image adaptation methods through transcording, aiming to reduce the size of images while maintaining their semantic value. Han et al. present an image adaptation method that involves several steps. ${ }^{11}$ First, border regions of an image that do not contain relevant semantic content are identified. Cropping is then used to remove irrelevant regions. Face detection, text detection, and contrast based saliency maps will be used to identify attention objects and combine them into a region of interest in the second step.

There has been some preliminary research on context-aware multimedia adaptation for mobile devices. For example, Jalal et al. propose a context-aware power-saving educational multimedia adaptation mechanism. ${ }^{12}$ In their approach, entire multimedia content is fragmented with multiple quality versions. Minimum quality constraints are determined and placed on each fragment. A fragment will be assigned with a higher quality presentation constraint if it has quality sensitive visual content. Multiple versions of the original content will enable a system to offer battery efficiency to different levels based on user preferences. The degree of power saving desired by a user determines the fragments to be selected and their individual quality. Yoo et al. propose a device capability-based adaptive streaming method for resource utilization efficiency. ${ }^{13}$ The real-time adaptive streaming method customizes streaming content among heterogeneous devices. The method is dependent on a device-capability based transcoding server for content transformation and has a choice of the number of transform daemons in accordance with the types of devices.

In summary, although there has been increasing research on multimedia content adaptation for mobile devices, most previous 
studies focused on video adaptation based on network traffic and bandwidth (i.e., network context). There are several limitations of existing approaches. First, there is no systematic approach that takes multiple different types of context into consideration simultaneously. Many studies adapt multimedia content based on a single type of context (e.g., network traffic). Second, most existing approaches use only one adaptation method, instead of a variety of adaptation methods for different situations. Third, while there has been some preliminary research on adapting multimedia content to fit the characteristics of mobile devices or network bandwidth, user adaptation preferences and accessibility needs have been rarely considered. For example, when a blind user browses a web page, he/she is not able to watch the visual content of a video file included in a web page. From a multimedia adaptation perspective, this is a user context that presents an opportunity to optimize network bandwidth utilization by removing the visual component and just delivering the audio signal of video to the user. It can help improve the overall user web browsing experience on mobile phones. Unfortunately, such user context has not been explored in multimedia adaptation for mobile Web. Last but not the least, most existing approaches offer a one-size-fits-all type of solution, rather than personalized solutions that satisfy individuals' adaptation preferences and needs.

\section{CADAMA: A Context-Aware and Dynamic Approach to Multimedia Adaptation}

\subsection{Architecture of CADAMA}

To address the limitations of existing methods, this study proposes a Context-Aware, Dynamic Approach to Multimedia Adaptation (CADAMA) (Figure 1) for mobile Web according to specific user and device context. User context includes user adaptation preference and possible special accessibility needs. Device context refers to the capability of multimedia support of individual mobile phones. We have developed a prototype system for Android phones to dynamically collect and analyze context and adapt multimedia content in websites accordingly. Such incorporation of user preferences enables a user-centered adaptation process.

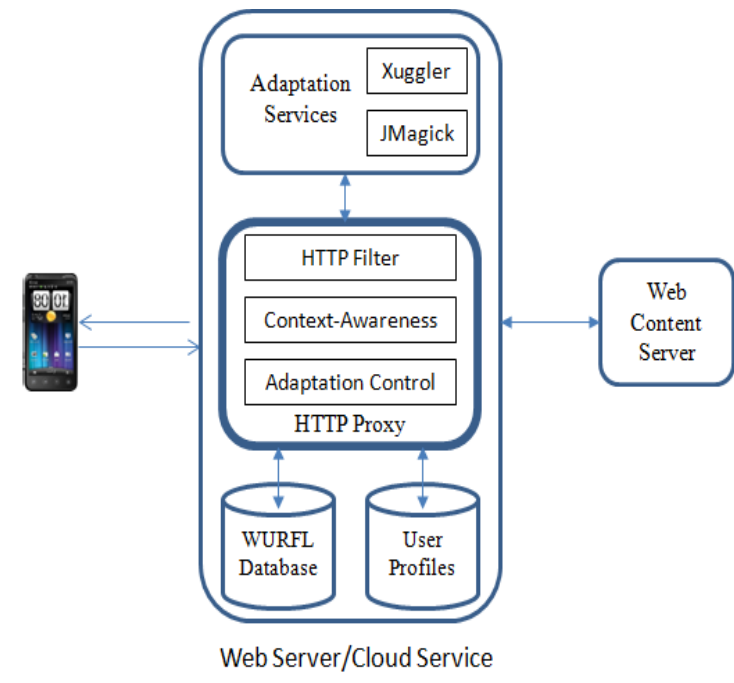

Fig. 1. The Overall Architecture of CADAMA

CADAMA is independent of mobile devices. There is no need to install any software on mobile devices when using its multimedia adaptation services. To implement multimedia adaptation, CADAMA first extends the HTTP proxy functionality by using an HTTP filter to process specific HTTP transactions. The filter searches for and extracts multimedia content from web content, if adaptation is needed, and passes it to an adaptation engine for processing. Once all adaptations are completed, web pages with adapted multimedia content will be sent to a user's mobile device.

An HTTP proxy is the central component of CADAMA. It controls adaptation process and coordinates executions of adaptation. As far as adaptation of streaming media (e.g., streaming video) is concerned, CADAMA currently supports progressive download of video, in which a media file is downloaded similar to the FTP mechanism over HTTP. A users' device buffers a downloaded video with a sufficient amount to start the playback. While playback is in progress, the remaining part of video will continue to be downloaded simultaneously.

\subsection{User-Centered Adaptation}

Each user has his/her profile stored in a database on a web server. It includes a user's accessibility needs (e.g., blindness) and user adaptation preferences in terms of what adaptations are preferred under different circumstances (e.g., remove images from a web page if a user is completely blind). For example, when blind users browse web pages on their mobile phones, they can't see enclosed images or visual playback of video. Therefore, CADAMA, by default, can automatically remove images and the visual 
component of video files, if any, after parsing the HTML code of a web page. This conserves wireless network bandwidth, which may also be preferred by a normal sighted user. Another example is that a color blind user (e.g., Protan) can't differentiate some colors as a normal sighted user can. As a result, an image could be unidentifiable for a color blind user when it contains colors that he can't distinguish. In such a case, CADAMA uses an optimization approach to adapting such images by replacing undifferentiable colors with differentiable ones while optimizing naturalization (i.e., closeness to the original colors) and differentiability.

Although CADAMA has default adaptation settings for different context, one of its major strengths lies in potential personalization of multimedia content adaptation from a user point of view. It is achieved by allowing users to specify their personal adaptation preferences in their user profile at any time through a GUI, which will overwrite default adaptation settings. When the HTTP proxy intercepts HTTP requests sent from a user device, it performs user authentication by matching the user name and password with information in user profiles, and retrieves accessibility needs and adaptation preferences from his/her profile. Those individual needs and preferences will be used by the proxy to determine actual adaptations that should be performed for the current user.

\subsection{Device-aware Adaptation}

Different mobile phones may differ in their hardware and software capabilities of supporting multimedia content. For example, certain audio or video formats may not be supported by a specific mobile phone. As a result, CADAMA needs to understand device capabilities of multimedia support before determining appropriate adaptations.

When the HTTP proxy receives an HTTP request, in addition to user authorization, it also parses the request to identify and extract information about a user's mobile device through a User-Agent header within the request. A UserAgent header contains information about the Web browser, the operating system, and hardware information of a mobile phone. If a requested web page contains multimedia content, CADAMA will access WURFL (http://wurfl.sourceforge.net/), a Device Description Repository (DDR), to derive the features of the user's mobile phone at run time. WURFL is a global database of thousands of mobile phones manufactured and represents a large, incremental matrix of devices and capabilities. For example, given the following information contained in a User-Agent header,
"Mozilla/5.0 (iphone; U; CPU iPhone OS 4_0 like Mac OS X; en-us) AppleWebKit/532.9 (KHTML, like Gecko) Version/4.0.5 Mobile/8A293 Safari/6531.22.7”, the system recognizes a mobile phone as an iPhone 4 with a mobile Safari 4 . More than five hundred features (i.e., capabilities) of a phone can be retrieved from WURFL. Due to the focus of this study on multimedia content adaptation for mobile Web, only certain capabilities of a phone that are related to multimedia support will be retrieved from WURFL. Then, the formats of multimedia content in a requested web page will be compared with the multimedia support capabilities of a mobile phone. If they do not match, the content needs to be adapted by converting the current incompatible media format into one that can be supported by the phone. Then the proxy will arbitrate and invoke corresponding adaptation services to perform specific multimedia content adaptation tasks based on built-in logics.

\subsection{Proxy}

The proxy adopts a Java based modular design consisting of multiple modules. It consists of several main components (Figure 2), which will be introduced in the rest of this section.

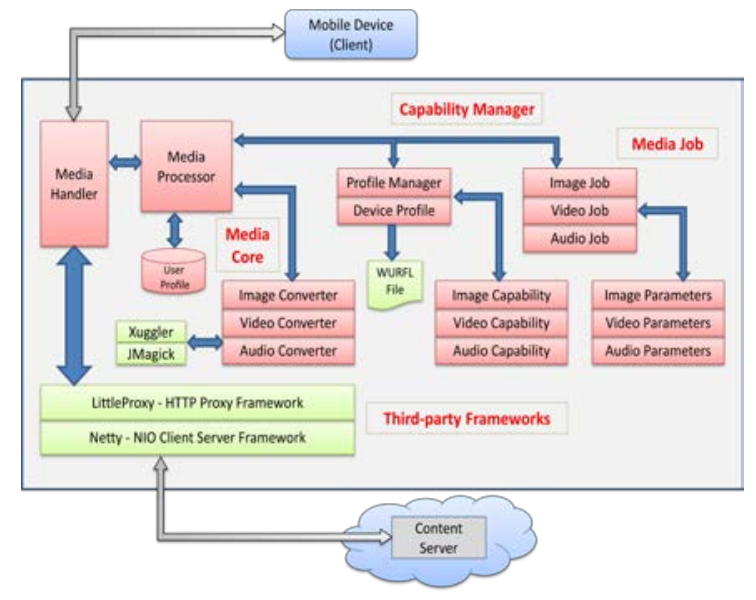

Fig. 2. The Architecture of Proxy

Media Core is a central control unit that makes various decisions for achieving a media adaptation goal. It is a logical module comprising of multiple components, including a Java package, a media handler, and a media processor. Media core performs HTTP traffic interception and extracts multimedia content. In conjunction with Capability Manager, it identifies multimedia content that needs to be processed according to the current device's multimedia support capabilities and performs content adaptation.

Media core interfaces with a user profile repository to acquire user adaptation preferences 
and accessibility needs. It facilitates personalized adaptation, coupling important user context with device-specific context. It interfaces with media processing services from third-party libraries.

Within Media Core, the Media Handler implements LittleProxy and hooks into the proxy framework to intercept HTTP traffic. LittleProxy is a high-performance HTTP proxy written in the Java Netty framework. The Media Handler invokes the Media Processor module.

The Media Processor performs multimedia inspection in two ways. It checks the value of a Content-Type HTTP header and identifies media type. In some cases, especially with video, a content server may not send relevant values in the Content-Type header. In such cases, knowing the exact format of video requires downloading the content and using third-party APIs to analyze content and find out content type. We improved this process by inspecting the header of multimedia content and metadata, instead of downloading the entire content. Once the content type is identified, Media Processor, with help from Capability Manager, acquires multimedia capabilities of the device requesting for the multimedia content and examines if the device supports the content (e.g., if device supports the codec required for video decoding). In addition, Media Processor also searches in the user profile repository for user preferences and accessibility needs (e.g., blind users), which brings the "User Context Awareness" into adaptation by transforming multimedia content in a way that meets users' preferences and needs.

Capability Manager provides a service for identifying multimedia capabilities of a specific device. Based on User-Agent information extracted from an HTTP request, Capability Manager searches the WURFL repository and retrieves technical specifications of the device. As introduced earlier, given the focus of this approach, Capability Manager is only interested in multimedia support properties of a device, such as supported codec types of audio, video, and image files.

Multimedia adaptation services in CADAMA are built based on off-the-shelf libraries including Xuggler and JMagick. Xuggler is a Java wrapper over a video processing tool called FFmpeg. It provides a rich set of APIs that can be easily used by a Java application and also provides a lowlevel Java abstraction of different FFmpeg constructs that are useful in decoding and encoding of video containers as well as streaming data. Xuggler provides two levels of APIs: simple APIs for encoding and decoding audio and video, and advanced APIs that provide control over encoding and decoding audio and video, as well as stream-level processing capabilities. Xuggler APIs provide an interface to detect the type of codec or format being used in video encoding, the size of the video, and frame rate, etc., which will then be used in adaptation. JMagick is an open source Java interface of the image processing library of ImageMagick. It provides an interface to detect the type of codec or format being used in image encoding, the file size of an image, and the width and length of an image.

Currently, CADAMA is able to perform the following multimedia adaptations automatically for web pages:

- Video adaptation (Figure 3):

1 ) Video format conversion (i.e., converting video from an incompatible format to a compatible format that can be supported by a specific mobile phone)

2) Visual signal removal from video

- Audio: audio format conversion

- Image:

1) Image removal (e.g., for a blind user)

2) Image resizing to fit screen dimensions of mobile devices (i.e., maximum width and height)

3) Image conversion (i.e., converting images from an incompatible format to a compatible format)

4) Image re-coloring for color-blind users. ${ }^{14}$

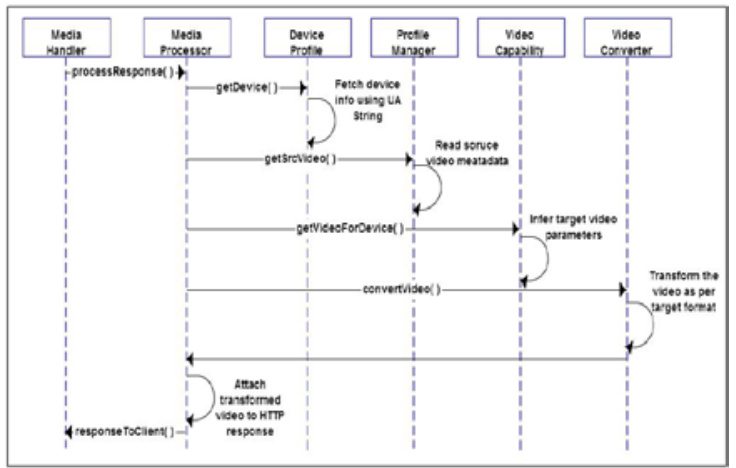

Fig. 3. Process Flow of Video Adaptation

\section{Evaluation}

We have evaluated the proposed CADAMA in terms of accuracy (i.e., whether or not the desired adaptation is performed properly in different contexts) and efficiency (i.e., how long an adaptation process takes).

We collected a dozen of websites that included different types of multimedia content from public domains (e.g., The New York Times). We also tested CADAMA for generalizability with some 
video samples collected from youtube.com because YouTube's content delivery protocol is different from that of many other websites. When YouTube delivers video content over HTTP, it uses proprietary headers and a generic content type instead of the content type specific to an actual medium. Formats of video contained in the selected websites include FLV (i.e., flash video) and MP4. As far as image formats, the selected websites included BMP (i.e., Bitmap Image File), GIF (i.e., Graphics Interchange Format), JPEG, and PNG (i.e., Portable Network Graphics), which are commonly seen image formats on the Internet. Audio formats included in the websites were MP3 and WAV (Waveform Audio File, a Microsoft and IBM audio file format standard).

All tests and measurements were done using a real Android mobile device instead of an emulator. That device supports some of the multimedia formats in selected websites, but does not support FLV video, bmp images (see Figure 4), and wav audio files. According to WURFL, the maximum viewable image width and height allowed by that device are $240 * 320$. The device's web browser was configured with the HTTP proxy hostname, and the port number was set to be 8080 , ensuring that all HTTP traffic would be routed via the configured HTTP proxy server. The logic built into this proxy performs content inspection and adaptation operations based on device characteristics and user needs/preferences.

\begin{tabular}{|l|l|}
\hline Device IMAGE property/feature & Value \\
\hline jpg & TRUE \\
\hline webp_lossy_support & TRUE \\
\hline gif & TRUE \\
\hline transparent_png_index & TRUE \\
\hline wbmp & TRUE \\
\hline webp_lossless_support & TRUE \\
\hline transparent_png_alpha & TRUE \\
\hline png & TRUE \\
\hline greyscale & FALSE \\
\hline epoc_bmp & FALSE \\
\hline bmp & FALSE \\
\hline gif_animated & FALSE \\
\hline svgt_1_1_plus & FALSE \\
\hline svgt_1_1 & FALSE \\
\hline tiff & FALSE \\
\hline colors & 65536 \\
\hline & \\
\hline
\end{tabular}

Fig. 4. Device Image Properties

We created user profiles for blind users and normal sighted users with different adaptation needs and preferences. For example, the profile of a blind user specifies the preference of removing visual component from video in websites. Then we tested CADAMA with the selected websites using those different user profiles. Results are shown in Table I. The columns in Table I represent the following:

- Adaptation type: the type of potential adaptation of multimedia content examined.

- Original format: the multimedia format before adaptation.

- Output format: the multimedia format generated after adaptation.

- Actual adaptation performed. There might be more than one adaptation process performed.

- Original media size: the size of multimedia content contained in a webpage before any adaptation. This is used as a reference to check how media size may change after adaptation.

- Post-adaptation size: the size of multimedia content after adaptation is performed. If adaptation is not performed, the size should not change from the original medium size.

- Processing time: the time taken to adapt multimedia content. It includes time taken to store a medium as the adaptation engine requires the complete file for processing.

As shown in Table I, we used 6 different websites for image adaptation testing. The first three contained images in .bmp format, which is not supported by the Android device used in evaluation. In addition, the size of original images exceeded the maximum width and height of the device. CADAMA properly performed two types of adaptation on the images in the first three websites, including image conversion and resizing, for normal sighted users, as shown in Table I. For other three websites that contained images in .gif, .jpg, and .png formats, because they were supported by the device, only resizing adaptation was performed. The adapted image dimensions have to be in proportion to the aspect ratio of an original image. For blind users that specified a preference of image removal, all those images were correctly removed.

Websites \#7 9 were used to assess video adaptation by removing the visual component of video for blind users. Websites \#7 and 8 contained video in FLV format, which was not supported by the device. Therefore, CADAMA removed the visual signal from the video and then converted it from FLV to a supported format (i.e., mp4). Because Website \#9 contained video in a supported format (i.e., mp4), only visual component removal adaptation was performed but no format conversion was required. 
Websites \#10 12 were also used to test video conversion adaptation. Website \#10 contained video in mp4 format, which is supported by the testing device. CADAMA correctly identified it and thus did not perform any adaptation. Websites \#11 and 12 contained video in the FLV format. Because it is not supported by the testing device, CADAMA adapted video in both websites by converting it from FLV to mp4 format.
Finally, we used websites \#13 15 to evaluate audio adaptation. The audio contained in Website \#13 was in the mp4 format supported by the testing device, so CADAMA did not perform any adaptation. Because audio contained in websites \#14 and 15 was in the wav format, which is not supported, CADAMA adapted audio by converting it from the wav format to the mp3 format.

Table I. Results of Evaluation

\begin{tabular}{|c|c|c|c|c|c|c|c|}
\hline $\begin{array}{c}\text { Adaptation } \\
\text { Type }\end{array}$ & $\begin{array}{c}\text { Sample } \\
\#\end{array}$ & $\begin{array}{c}\text { Original } \\
\text { Format }\end{array}$ & $\begin{array}{l}\text { Output } \\
\text { Format }\end{array}$ & $\begin{array}{c}\text { Adaptation } \\
\text { Performed }\end{array}$ & $\begin{array}{l}\text { Original Size } \\
\text { (Bytes) }\end{array}$ & $\begin{array}{c}\text { Post-Adaptation } \\
\text { Size }\end{array}$ & $\begin{array}{l}\text { Processing } \\
\text { Time (ms) }\end{array}$ \\
\hline \multirow{6}{*}{$\begin{array}{c}\text { Image } \\
\text { Conversion }\end{array}$} & 1 & bmp & jpg & $\begin{array}{l}\text { YES (1) Convert } \\
\text { format (2) Resize }\end{array}$ & $\begin{array}{l}2,359,350 \\
(1024 \times 768)\end{array}$ & $9,187(240 * 180)$ & 139 \\
\hline & 2 & bmp & jpg & $\begin{array}{l}\text { YES (1) Convert } \\
\text { format (2) Resize }\end{array}$ & $\begin{array}{c}786,486 \\
(512 \times 512)\end{array}$ & $\begin{array}{c}11,944 \\
(240 * 240)\end{array}$ & 44 \\
\hline & 3 & bmp & jpg & $\begin{array}{l}\text { YES (1) Convert } \\
\text { format (2) Resize }\end{array}$ & $\begin{array}{c}385,254 \\
(300 * 428)\end{array}$ & $\begin{array}{c}21,486 \\
(224 * 320)\end{array}$ & 41 \\
\hline & 4 & gif & gif & $\begin{array}{c}\text { Yes } \\
\text { (Resize) }\end{array}$ & $\begin{array}{c}182,701 \\
(515 * 480)\end{array}$ & $\begin{array}{c}31,648 \\
(240 * 224)\end{array}$ & 222 \\
\hline & 5 & jpg & jpg & $\begin{array}{c}\text { Yes } \\
\text { (Resize) }\end{array}$ & $\begin{array}{c}268,729 \\
(1600 * 1200)\end{array}$ & $\begin{array}{c}23,632 \\
(240 * 180)\end{array}$ & 256 \\
\hline & 6 & png & png & $\begin{array}{c}\text { Yes } \\
\text { (Resize) }\end{array}$ & $\begin{array}{r}2,439,708 \\
(1922 \times 1082)\end{array}$ & $\begin{array}{c}51,436 \\
(240 * 135)\end{array}$ & 275 \\
\hline \multirow{3}{*}{$\begin{array}{l}\text { Stripping } \\
\text { Video }\end{array}$} & 7 & FLV & mp4 & $\begin{array}{c}\text { Yes (removal + } \\
\text { Conversion) }\end{array}$ & 88,722 & 100,101 & 379 \\
\hline & 8 & FLV & mp4 & $\begin{array}{c}\text { Yes (removal + } \\
\text { Conversion) }\end{array}$ & 129,444 & 273,414 & 757 \\
\hline & 9 & mp4 & mp4 & Yes & $3,144,689$ & 131,476 & 1,047 \\
\hline \multirow{3}{*}{$\begin{array}{c}\text { Video } \\
\text { Conversion }\end{array}$} & 10 & mp4 & mp4 & No & 866,964 & 866,694 & N/A \\
\hline & 11 & FLV & mp4 & Yes & 88,722 & 102,760 & 272 \\
\hline & 12 & FLV & mp4 & Yes & 129,444 & 548,387 & 919 \\
\hline \multirow{3}{*}{$\begin{array}{c}\text { Audio } \\
\text { Conversion }\end{array}$} & 13 & mp3 & mp3 & No & 933,012 & 933,012 & N/A \\
\hline & 14 & wav & mp3 & Yes & 304,578 & 27,828 & 174 \\
\hline & 15 & wav & mp3 & Yes & 41,644 & 7,766 & 45 \\
\hline
\end{tabular}

\section{Conclusion}

This study presents CADAMA, a user-centered, device-aware, and dynamic adaptation approach to multimedia adaptation for mobile Web. The proposed approach uniquely takes users' adaptation preferences and personal needs into consideration, and enables adaptation based on multimedia support capabilities of specific mobile handheld devices that users are using. The decision and execution of adaptation are made dynamically at run time instead of pre-determined in a fixed manner. The preliminary evaluation of system performance showed that CADAMA could accurately determine and execute appropriate adaptations under different contexts, and processing time for adaptation is reasonable.

In general, adaptation time tends to increase as the size of video, audio, and image grows. This problem can be alleviated by performing real-time optimization and adaptive streaming. For example, with adaptive streaming, a video file will be segmented into short video chunks of small size. Adaptation can be performed on those small video chunks individually to shorten the response delay. Another potential solution to improving adaptation efficiency is to pre-fetch the most popular content ahead of time for a specific user if his/her interest in this content may be known a priori or inferred from 
his previous browsing history. ${ }^{15}$ As a result, multimedia adaptation can be performed in advance.

In the current design, when a given device supports more than one codec, CADAMA selects a target codec based on a set of heuristics. In some situations, the medium size after adaptation may be larger than that of an original medium. In future research, we will refine and optimize target codec selection by choosing the most efficient and cost-effective codec among all alternatives supported by a device. We also plan to conduct a user study in the future by asking participants to use CADAMA while browsing websites with multimedia content and collecting their perception and feedback to further improve the approach.

There are other possible contexts that we plan to explore and incorporate into the current CADAMA system, such as network context. The gap between traffic demand and the wireless link capacity, along with real-time link conditions, results in poor service quality of video streaming over mobile networks, such as long transmission and buffering time. ${ }^{16,17}$ Variations in wireless network bandwidth during a user's browsing session may also be utilized to improve user experience. When available bandwidth drops below a certain threshold due to heavy traffic, adaptation can be performed by either removing multimedia content from a webpage because of its considerable size, or converting multimedia content into a lower (yet acceptable) resolution/quality, in order to reduce the demand on the network bandwidth and shorten the transmission delay.

It is also important to note that a web page is fetched in pieces by the HTTP proxy independently. Adaptation of multimedia content is a CPU intensive operation. Because the HTTP proxy server is multi-threaded, every medium part (e.g., image or video) is processed in parallel in CADAMA. Thus the overall processing time of a web page is influenced by the processing power of the proxy server.

\section{ACKNOWLEDGMENT}

This material is based upon work supported by the U.S. National Science Foundation (IIS-1250395). Any opinions, findings, and conclusions or recommendations expressed in this material are those of the authors and do not necessarily reflect the views of the National Science Foundation.

\section{REFERENCES}

[1] D. Zhang, and J. Lai, "Can convenience and effectiveness converge in mobile web? A critique of the state-of-the-art adaptation techniques for web navigation on mobile handheld devices." International Journal of HumanComputer Interaction, 27(12), 2012, pp.1133-1160.

[2] D. Zhang, "Web Content Adaptation for Mobile Handheld Devices." Communications of the ACM (CACM). 50(2), 2007, pp. 75-79.

[3] D. Kim, D. Yun, and K. Chun, "Context-aware adaptive streaming in Web of objects environments.” Proceedings of 2014 IEEE World Forum on Internet of Things. Soeul, Korea. March 6-8, 2014. pp.63-64

[4] H. Louafi, S. Coulombe, and U. Chandra, "Quality preduction-based dynamic content adaptation framework applied to collaborative mobile presentations." IEEE Transactions on Mobile Computing. 12(10), 2013. pp. 2024-2036.

[5] D. Zhang, B. Adipat and Y. Mowafi, "User-Centered Context-Aware Mobile Applications-The Next Generation of Personal Mobile Computing." Communications of the AIS. 24, 2009, pp.27-46.

[6] G. Tian and Y. Liu, "Towards agile and smooth video adaptation in dynamic HTTP streaming.” Proceedings of the $8^{\text {th }}$ International Conference on emerging Networking Experiments and Technologies. 2012, pp.109-120.

[7] S. Bruyne, P. Hosten, C. Concolato, M. Asbach, J. Cock, M. Unger, J. Feuvre, and R. Walle, "Annotation based personalized adaptation and presentation of videos for mobile applications. Multimedia Tools and Applications.” 55(2), 2011, pp.307-331.

[8] G. Abebe, D. Coquil, and H. Kosch, "Enhancing semantic video adaptation speed through compressed domain." Woskshop on Multimedia on the Web. September 8, 2011, pp.43-44.

[9] I. Kofler, R. Kuschnig, and H. Hellwagner, "Innetworking real-time adaptation of scalable video content on a wifi router." Proceedings of the $6^{\text {th }}$ IEEE Consumer Communications and Networking Conference. Jan. 1013, 2009

[10] W. Yin, J. Luo, and C. Chen, "Event-based semantic image adaptation for user-centric mobile display devices.” IEEE Transactions on Multimedia. 13(3), 2011, pp.432-442.

[11] R. Han, P. Bhagwat, R. Lamaire, T. Mummert, V. Perret, and J. Rubas, "Dynamic adaptation in an image transcoding proxy for mobile web browsing." IEEE Personal Communications. December, 1998, pp.8-17.

[12] S. Jalal, N. Gibbins, D. Millard, B. Al-Hashimi, and N.R. Aljohani, "Context-aware power saving multimedia adaptation for mobile learning. Proceedings of 2013 Seventh International Conference on Next Generation Mobile Apps, Services and Technologies. pp. 256-261

[13] Y. Yoo, I. Lee, and Y. Chung, "Real-time adaptive streaming machanism with device capabiity for resource efficiency." Proceedings of Workshop on Computing, Networking and Communcations. 2012.pp. 204-210.

[14] L. Zhou, V. Bansal, and D. Zhang (2014). Color Adaptation for Improving Mobile Web Accessibility. The 13th IEEE/ACIS International Conference on Computer and Information Science. June 4-6, 2014, Taiyuan, China. p.291-296. 
[15] A. Devlic, S. Kista, P. Lungaro, P. Kamaraju, Z. Segall, "Energy consumption reduction via context-aware mobile video pre-fetching." Proceedings of IEEE International Symposium on Multimedia. Irvine, CA, USA. Dec. 1012, 2012, pp.261-265.

[16] Chen, M. “AMVSC: a framework of adaptive mobile video streaming in the cloud”. Proceedings of Globecom 2012-Communications Software, Services and Multimedia Symposium. pp. 2042-2047.

[17] Miller, K, Quacchio, E., Gennari, G., and Wolisz, A. "Adaptation algorithm for adaptive streaming over HTTP." Proceedings of 2012 IEEE $19^{\text {th }}$ International Packet Video
Workshop. May 10-11, 2012, Munich, Germany. pp.173178 